\title{
Proceedings of the meeting of the Association of British Neurologists held in Newcastle upon Tyne, 3-4 April 1987
}

\begin{abstract}
DEFECTS OF FATTY ACID OXIDATION IN SKELETAL MUSCLE

DM Turnbull, B Ashworth, K Bartlett, I Shepherd. Departments of Neurology and Child Health, University of Newcastle upon Tyne and Department of Neurology, Northern General Hospital, Edinburgh
\end{abstract}

Fatty acids are important substrates for both exercising and resting skeletal muscle. Impaired fatty acid oxidation in human skeletal muscle causes weakness and/or pain associated with lipid accumulation in muscle fibres. Fatty acid oxidation is a complicated process involving at least 12 enzymes and there are many potential sites at which a defect may occur. Many cases of lipid storage myopathy were thought to be due to carnitine deficiency although this is now thought to be unlikely in the majority of cases. The most probable site of the enzyme abnormality in these patients is a defect of one of the three acyl-CoA dehydrogenases, the group of enzymes at the beginning of the B-oxidation sequence. We describe a 38 year old male who presented with a painful proximal myopathy whose muscle contained an abnormal accumulation of lipid. The activity of both short- and medium-chain dehydrogenase was low in this first biopsy. The patient improved clinically and electrophysically with the administration of riboflavin, and a repeat muscle biopsy revealed only a minor morphological abnormality as well as improved activity of the acyl-CoA dehydrogenases. This unique case highlights the importance of comprehensive investigation of patients with lipid storage myopathies, since these disorders are potentially treatable.

\section{THE ANALYSIS OF NEUROMUSCULAR}

TRANSMISSION IN MOTOR NEURON DISEASE

TJ Walls, CR Slater, PRW Fawcett, IS Schofield. Muscular Dystrophy Laboratories, Newcastle General Hospital, Newcastle upon Tyne.

Single fibre EMG reveals defects of neuromuscular transmission in several neuromuscular diseases where the principal abnormality is not at the neuromuscular junction. These include motor neuron disease, spinal muscular atrophy and the muscular dystrophies. The pathophysiology of these transmission defects is unknown.To examine their cellular basis, we have developed procedures for studying the structure and function of motor innervation in motor point biopsies from the vastus lateralis.

In addition to routine histological and histochemical examination, quantitative morphological studies of the intramuscular nerves and neuromuscular junctions are made. To study function, nerve-muscle preparations are obtained from portions of the biopsy and intracellular recording techniques are used to study transmitter release and action.

We have investigated 21 patients with a variety of myopathic and neurogenic diseases so the number of patients with any given condition is still small. However, our results from patients with motor neuron disease already suggest that in this condition there is a reduction in the amount of transmitter released from the nerve terminals at some neuromuscular junctions. If this occurs in vivo, it would reduce the safety factor at these junctions and might account for the increased jitter and impulse blocking on single fibre EMG.

\section{MYOTOXIC EFFECTS OF KRAIT VENOMS}

BA Summers, JB Harris. Muscular Dystrophy Group Research Laboratories, Department of Neurology, University of Newcastle, Newcastle upon Tyne

Snake bite is a common cause of distress. Although krait venom is generally considered purely neurotoxic, a clinical report of five bites by the Malayan krait, Bungarus candidus, included a case with generalised muscle tenderness and pain on passive movement. This raised the possibility of myotoxicity which was assessed using the rat soleus muscle. Crude venoms from four krait species were injected subcutaneously into hind limbs of anaesthetised rats. Soleus muscles were removed between 1 hour and 7 days later and frozen in Arcton. Polyvalent and monovalent antivenoms were examined.

The venom of Bungarus candidus caused a dose dependent muscle necrosis with mores than $75 \%$ of muscle fibres destroyed by 0.40 $\mathrm{mg} / \mathrm{kg}$ body weight. Muscle fibre degener $\frac{\mathrm{\sigma}}{0}$ ation and infiltration by inflammatory cells was seen at 6 hours and appeared maxima 24 hours after injection. Muscles sampled a€ 1 week showed muscle fibre regeneration Comparable results were obtained using $B-$ fasciatus venom, but no such effect was seen with venoms of $B$ caeruleus or $B$ mult $i \overrightarrow{\vec{\omega}}$ cinctus. Calculations based on venom yieldo suggest some relevance for human tox icology; an average adult specimen of $B$ fas $\frac{\bar{O}}{0}$ ciatus yields $25-50 \mathrm{mg}$ venom. Inoculationic of $25 \mathrm{mg}$ into a $60 \mathrm{~kg}$ man is equivalent to $\mathrm{a}$. dose of $0.41 \mathrm{mg}$ crude venom $/ \mathrm{kg}$ body $\overrightarrow{\mathrm{N}}$ weight delivered locally.

IMMUNOLOGICAL OBSERVATIONS ON PARANEOPLASTIC SENSORY NEUROPATHY DJ Dick, JB Harris, JB Foster. Muscuf Dystrophy Laboratories, Newcastle Genexal Hospital, Newcastle upon Tyne

We have identified three patients with paraneoplastic sensory neuropathy assoofated with a small cell carcinoma of lung. $\overrightarrow{0}$ Two were plasmaphoresed but showed no clinical improvement. Serum from all three patients contained an IgG antibody whicho bound to neuronal nuclei in dorsal root ganglia, spinal cord and cerebral cortex. Nucleio in non-neural tissues were negative. The $\mathrm{IgG}_{2}^{\circ}$ contained both kappa and lambda light $\stackrel{\odot}{\varnothing}$ chains and could fix complement. The antibody also reacted with dorsal root ganglia윽 from rodents. Western blotting techniques have demonstrated antibody binding to a constant and specific band.

A passive transfer of the neuropathy was attempted by injecting a cohort of mice with plasma from an affected patient for $100 \%$ days. No clinical or morphological effect was 3 observed and no human immunoglobulin was found in the mouse dorsal root ganglia. $\frac{\text { O }}{3}$ We have however demonstrated that the an- $₹$ tibody can enter neurons and bind to neu-을 ronal nuclei culture although it does not $D$ appear to alter their viability. It is still un-음 clear from these results whether the antibody is directly involved in the pathogenesis of $0 \mathrm{r}$ this disease. 
SHORT TERM STABILITY OF SINGLE MOTOR UNIT RECORDINGS IN MOTORNEURONE DISEASE. A MACRO EMG STUDY

RJ Guiloff, H Modarres-Sadeghi, H Rogers. Department of Neurology, Westminster Hospital, Charing Cross and Westminster Medical School, London

We have previously shown an acute $25-30 \%$ increase in mean corrected fibre density and in mean Macro EMG median amplitude and area of brachial biceps in 29 patients with MND receiving a TRH analogue (RX77368).

Baseline Macro EMG recordings of seventeen voluntarily activated single motor units and five fasciculations in nine MND patients showed a small mean \% standard error for both mean amplitude $(2.38 \%$, range $0.58-8.84)$ and mean area $(2.62 \%$, range $0 \cdot 25-5 \cdot 95$ ). Recordings every 15 minutes for 2 hours of single motor units, with ( 11 units) and without ( 11 control units) intravenous infusion of $0.2 \mathrm{mg} / \mathrm{kg}$ of RX77368, showed no significant change in amplitude nor area with the drug.

The findings suggest that with this technique, over short periods, no gross change in the functional peripheral territory of individual motor units in the muscles studied can be detected during drug administration. The results are consistent with the hypothesis that the effect on fibre density and Macro EMG previously found may relate to direct or indirect action of RX77368 on anterior horn cells.

A NEW PORTABLE UNIT FOR THE ASSESSMENT OF THERMAL SENSATION PATHWAY IN MAN GA Jamal, S Hansen, Al Weir, JP Ballantyne. Department of Neurological Sciences, St. Bartholomew's Hospital, London and Institute of Neurological Sciences, Glasgow

A new microprocessor controlled, self contained, portable and easy to operate unit for the assessment of the small diameter afferent thermal pathways with high sensitivity and reproducible is described. The technique uses the Peltier effect, the two alternative forced-choice method of psychophysical analysis, the up-and-down transform rule of mathematical assessment and standardises all other sources of variability.

The method tests the functional integrity of the thinly myelinated(A $\triangle)$ and unmyelinated $(\mathrm{C})$ fibres which cannot be assessed by conventional nerve conduction and EMG and somatosensory evoked potential studies. From the application of the technique to a large group of patients with peripheral neuropathy of various aetiology, it is concluded that it is of value in (a) the early detection of subclinical and serial assessment of ongoing neuropathy, (b) reliable diagnosis of selective small fibre neuropathy, (c) serial monitoring of the effect of therapy on neuropathy, (d) the assessment of neurotoxic effects of various industrial products and pharmaceutical agents, and (e) detection of subtle sensory dysfunction in other conditions.

INVESTIGATION OF DEFECTS OF FATTY ACID OXIDATION IN SKELETAL MUSCLE DM Turnbull, K Bartlett, A Aynsley-Green, HSA Sherratt, NJ Watmough, I Shepherd, AKMJ Bhuiyan. Departments of Neurology, Child Health and Pharmacological Sciences, University of Newcastle upon Tyne

Myopathies due to defective fatty ocid oxidation are potentially treatable by dietary manipulation and/or drug therapy. It is therefore important that this group of disorders is properly investigated so that the site of the enzyme abnormality can be detected and appropriate treatment instituted. Many previous cases of lipid storage myopathy have only been studied by morphological examination of the muscle biopsy and determination of the carnitine concentration. Carnitine deficiency is a secondary biochemical change in the majority of patients. Our approach involves investigating the possibility of other organ involvement by measuring blood and urine metabolites during a prolonged fast. We determine the concentration of individual acyl carnitine esters in the blood and urine by hplc and this often suggests the site of the defect. We have shown that the radiochemical measurement of fatty acid oxidation in skeletal muscle is inadequately assessed by measuring radioactive $\mathrm{CO}_{2}$ release alone. We have developed a radio-hplc method to show that the majority of the reaction product is in the acid soluble fraction and is either citric acid cycle intermediates or acetylcarnitine. Assay of the individual enzymes of B-oxidation, in particular the acyl-CoA dehydrogenases, requires sensitive methods. Using an ETFlinked assay we can measure the activity of the three acyl-CoA dehydrogenases in $10 \mathrm{mg}$ of muscle. These techniques allow accurate assessment of fatty acid oxidation in skeletal muscle and help in the diagnosis of this important group of muscle diseases.

NORMAL AND PHYSIOLOGICAL VARIATIONS IN CRANICAL CSF VOLUME MEASURED BY MAGNETIC RESONANCE IMAGING

R Grant, B Condon, A Lawrence, D Hadley,
J Patterson, I Bone, GM Teasdale. Institute $\bar{Z}$ of Neurological Sciences, Glasgow

We have developed a method of measuring CSF volumes, using a new MRI sequence. The relationships between CSF volume, age, in sex difference, skull circumference and the menstrual cycle have been assessed.

Sixty-four normal subjects between the ages of 18-64 were studied. The pulse sequence used (IRCP 300/440/5000) provides a sagittal image of cranial CSF only. Total $\stackrel{\rho}{\rightarrow}$ cranial, cortical sulcal, ventricular and pos- 0 terior fossa CSF volumes were calculated.

Total cranial and ventricular CSF vol- $\bar{\omega}$ umes ranged from $57 \cdot 1-286 \cdot 5 \mathrm{mls}$ and 6.8-30 mls respectively (male mean $146 \mathrm{mls}$; female mean $114.5 \mathrm{mls}$ ). Total and cortical sulcal on volumes increased steeply with age in males $\overrightarrow{0}$ and females while ventricular and posterior fossa CSF volumes showed a less marked $\vec{\omega}$ increase with age and wide variation. There $\Omega$ was no association between skull circumference and total cranial CSF volume. Im- $\frac{7}{0}$ mediate reproducibility studies in 25 subjects $f$ demonstrated a median difference in ventricular and cortical sulcal volume of $0.2 \mathrm{mls}$ and $2.1 \mathrm{mls}$ respectively. Of 20 women with $\vec{V}$ a normal menstrual cycle, 19 had more tot욕 $\vec{\sigma}$ cranial CSF pre-menstrually than at miक cycle.

The validation of this technique allows the future study of CSF volumes in neurologica and neurosurgical conditions and asses ment of medical or surgical treatment.

TRANSIENT FEELINGS OF COMPULSION CAUSEO BY HEMISPHERIC LESIONS: THREE CASES CD Ward. Southampton University, Southampton

Feelings of compulsion are described in post-encephalitic states-where the pathology is poorly localised-and in Gilles de la Tourette syndrome, in which the pathological basis is poorly understood. In both conditions the ensuing involuntary move- 3 ment generally overcomes internal resistance and seems to be the primary phenomenon, whereas it has been suggested that in obsession-compulsion disorder the reverse is true. Three patients with no previous? psychiatric history described feelings of compulsion which were clearly associated with hemispheric lesions and which must o have been primary since no movement occurred. A woman of 59 presented with an 은 attack in which she successfully resisted a compulsion to walk to the left. A man of 43 음 repeatedly experienced a transient compulsion to move the right arm and to shout. $N$ These patients had hemispheric gliomas. A 
right-handed woman of $62 \mathrm{had}$ an episode of dysphasia which resolved within a few minutes. In the next few weeks she felt the need on 3 or 4 occasions to prevent her right arm from moving against her will. The only abnormality on CT scan was a dubious lucency in the left basal ganglia. These cases show that feelings of compulsion may arise as primary neurological disturbances rather than as epiphenomena of movement disorders.

THE NEUROLOGICAL CONSEQUENCES OF HUMAN IMMUNODEFICIENCY VIRUS INFECTION M Johnson, R Clifford Jones, SM Forster, AJ Pinching, JRW Harris. St. Mary's Hospital, Praed Street, London

Reports from USA indicate that as many as $30 \%$ of patients with human immunodeficiency virus (HIV) infection may have neurological problems in life, and that $80 \%$ have neuropathological abnormalities at autopsy. There are reasons to expect that the manifestations of this infection may differ from country to country, depending for example on the prevalence of local endemic infections. At St. Mary's Hospital, where the largest group of HIV + ve patients in this country have been cared for, we are rapidly accumulating cases in which neurological disturbances complicate this disease, and here report the salient features in the first 40 patients with neurological abnormalities The pattern broadly follows that described from the USA with the majority showing encephalopathic features. Our patients showed insidiously progressive dementia which has to be distinguished from other encephalopathies which may be reversible and from the effects of opportunistic infection. We have also studied a group with a painful, symmetrical, sensorimotor neuropathy, and describe one patient with proximal myopathy. The commonest opportunistic infections of the CNS have been toxoplasmosis (eight cases) and cryptococcosis (eight cases). The advent of treatment trials for HIV infection will demand reliable assessment of neurological involvement, and we will describe psychometric and electrophysiological approaches to this problem.

\section{MAGNETIC RESONANCE IMAGING AND} ELECTROPHYSIOLOGICAL STUDIES OF THE OPTIC NERVE IN OPTIC NEURITIS DH Miller (introduced), MR Newton (introduced, JC van der Poel (introduced), G Johnson (introduced), DG MacManus (introduced), AM Halliday, WI McDonald. Institute of Neurology, Queen Square, London
Using a newly introduced magnetic resonance imaging (MRI) sequence (STIR) we have studied the optic nerves in 33 patients aged 7-44 years with optic neuritis. Visual evoked potentials (VEP) were recorded in all cases.

Twenty seven had had unilateral optic neuritis and six bilateral. Of the 39 optic nerves clinically affected, MRI was abnor$\mathrm{mal}$ in 33 and VEP in 38 . Of 27 asymptomatic optic nerves, MRI was abnormal in three and VEP in four. The MRI lesions were in the anterior part of the nerve in 18 $(50 \%)$, in the middle in $25(72 \%)$, and in the posterior part in $(25 \%)$. Nine of 10 with disc swelling had a lesion visible at MRI; the lesion was anterior within the nerve in five, in the mid portion in three and posterior in one. All 15 with clinical evidence of optic atrophy had a positive MRI.

The mean length of lesions in patients with poor visual recovery (VA $>6 / 9$ ) was $18.6 \mathrm{~mm}$, and in those with a good recovery, $10.0 \mathrm{~mm}$. The six nerves where no abnormality was seen all had a good visual recovery.

There was no correlation of visual prognosis with the site of the lesion along the nerve. There were no particular VEP correlates with the site and length of MRI lesions.

VISUAL FUNCTION AND CEREBROSPINAL FLUID IMMUNOGLOBULINS IN EARLY MULTIPLE SCLEROSIS

WP Honan, JR Heron, DH Foster, MO Scase, GK Edgar. Department of Postgraduate Medicine, University of Keele, Department of Communication and Neuroscience, University of Keele

We report the initial findings in a longitudinal study of visual function and cerebrospinal fluid (CSF) immunoglobulins in multiple sclerosis. Cases were studied as near as possible to the time of clinical onset of the disease.

Visual function was assessed clinically and by visual fields, visual evoked potentials (VEP) and psychophysical measures of luminance and chromatic thresholds and the de Lange attenuation characteristic. CSF immunoglobulins were estimated using IgG levels, IgG synthesis and agar-gel electrophoresis.

Thirty two cases were included with an average symptom duration of 7.6 months, range $0.25-14$ months. The series was subdivided into two groups: A: without optic neuritis and B: with optic neuritis. Abnormalities of at least one measure of visual function were detected in $26 / 32(81 \%)$ cases; CSF immunoglobulins were abnormal in $19 / 31(61 \%)$ cases. Analysis of the sub- groups revealed abnormalities of visual $Z$ function in $13 / 18(72 \%)$ cases in group $A \stackrel{\mathbb{D}}{\complement}$ and $13 / 14(93 \%)$ cases in group B. CSF immunoglobulins were abnormal in $8 / 17^{\text {? }}$ $(47 \%)$ cases in group A and 11/14 (79\%) cases in group $\mathbf{B}$.

VISUAL FUNCTION IN PATIENTS WITH MULTIPLE SCLEROSIS AND NORMAL VISUAL ACUITY

B Ashworth, PA Aspinall, JD Mitchell. Department of Clinical Neurosciences, University of Edinburgh

Eighty nine patients with multiple sclerosis and normal visual acuity have been submit- $\overline{\bar{c}}$ ted to a battery of test of visual function. The $\overparen{\nabla}$ tests included the visual evoked potential, $\varrho$ tests of colour vision and lightness discrimi- صs nation, and spatial contrast sensitivity. The $\overrightarrow{-}$ Posner diagnostic classification was used. This group of controls and patients forms $\overrightarrow{\vec{\omega}}$ the basis of a continuing serial study over $\stackrel{\omega}{a}$ five years.

Results show significant difference be- $\frac{7}{0}$ tween the clinical and normal groups in of yellow-blue, lightness discrimination, and at several frequencies of spatial contrast sensi- $\vec{N}$ tivity in the range 0.3 to 10 cycles/degret $\overrightarrow{-}$ Abnormalities of these measures were der金 $\vec{\nabla}$ onstrated in patients with no history of acu optic neuritis. Further, more significar $\frac{1}{,}, \frac{}{\supset}$ differences between the clinically definit and probable groups were found in res green discrimination and contrast sens tivity.

LAMBERT-EATON MYASTHENIC SYNDROME: CLINICAL FEATURES AND RESPONSE TO

TREATMENT IN 50 CASES

JN O'Neill (introduced), NMF Murray, $\mathbf{J} \cdot$ Newsom-Davis. Institute of Neurology, Queen Square, London

The Lambert-Eaton myasthenic syndrome is a rare pre-synaptic disorder of neu- 응 romuscular transmission, often associated $\varrho$ with carcinoma of the lung, in which the $\overrightarrow{\overline{0}}$ number of acetylcholine packages released 3 by a nerve impulse is reduced. Recent experimental studies have shown that it has an autoimmune basis in both its cancerassociated and non-cancer-associated forms, the disorder of transmission being due to an? IgG antibody that interferes with the function of voltage-dependent calcium channels at motor nerve terminals. No large clinical o series has been reported apart from the description of electromyographic findings in $17 \mathrm{O}$ cases by Lambert et al in 1961.

The 50 consecutive cases seen by one of us $\frac{D}{2}$ (JND) over the last eight years formed the basis for this study. Electromyographic and $N$ autoantibody studies were undertaken in all

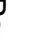
, 
patients. The disease was associated with overt malignancy in 23 cases, histologically proven to be small cell lung carcinoma in 20 disease duration in the remaining 27 cases ranged from one to twelve years. We have analysed the clinical features, course (monitored clinically and electromyographically) and response to treatment that included 3,4-diaminopyridine, immunosuppressive drugs, plasma exchange and, in cancer cases, chemotherapy.

MYELOGRAPHY IN A REGIONAL NEUROLOGY UNIT; USE, MISUSE AND COSTS

PAG Sandercock, LD Blumhardt, MA Roberts. Department of Neurology, Walton Hospital, Liverpool

The number of myelograms performed each year in our unit has increased steadily from 289 in 1972 to 612 in 1985 . We have audited our use of myelography over 6 months. We present data on the first 318 myelograms performed on 311 patients. The indication (some patients had more than one) for myelography was: lumbar or cervical radiculopathy in $170(55 \%)$, spinal cord lesion for $>1$ month in $85(27 \%)$, spinal cord lesion $<1$ month in $27(9 \%)$, suspected ArnoldChiari malformation in $22(7 \%)$, suspected spinal dysraphism $9(3 \%)$, other $85(27 \%)$. Ancillary investigations performed were: visual evoked potentials performed before the myelogram in 15 cases (five were abnormal), CT of the spine in 44 and CT of the head in 96 . Myelography had to be repeated in 25 patients (often because the test, performed elsewhere, was inadequate). Clinical information on X-ray request forms was inadequate or misleading in many cases. The radiology department costs were small in comparison to the hospital cost of $£ 130$ per bed day. The mean time from admission to myelography was 2.98 days. Several areas where our use of this test might be improved have been identified.

ILLNESS BEHAVIOUR AND PSYCHIATRIC DISEASE IN NEUROLOGICAL PATIENTS

RA Metcalfe, D Firth, SS Pollock, M Timol, $F$ Creed. Departments of Neurology and Psychiatry, Manchester Royal Infirmary

The relationship between neurological and psychiatric illness has previously been studied in those selected patients who have been referred to a psychiatrist. We have assessed a consecutive series of female patients admitted to a neurological investigation unit, to examine the likelihood that the patients' symptoms were explicable in terms of neurological and/or psychiatric illness. Of $93 \mathrm{pa-}$ tients 54 were thought by the neurologist to have symptoms that were entirely explicable in terms of organic disease (group 1). Twenty two had symptoms that were less clearly organic in origin (group 2), and in 17 the neurologist was sure that the symptoms could not be due to organic disease (group 3). The median total psychiatric score according to the Clinical Interview Schedule, was 13 for group 1, 25 for group 2 and 30 for group 3 (p 0.001). But the hypochondriasis score of the Illness Behaviour Questionnaire (phobic concern about health) was $0 \cdot 5,1$ and 5 for the three groups (p 0.001) indicating that illness behaviour was far more evident in group 3. Psychiatric illness could entirely explain the symptoms in only 2 of the group 2 patients and $8 / 17$ of the group 3 patients. It is suggested that this method of evaluation could indicate which patients with "functional" symptoms require psychiatric treatment and which require further investigation.

SUBACUTE ADMINISTRATION OF A TRH ANALOGUE (RX 77368) IN MOTOR NEURON DISEASE (MND). OPEN STUDY

H Modarres-Sadeghi (introduced), RJ Guiloff. Department of Neurology, Westminster Hospital, Charing Cross and Westminster Medical School, London

A controlled acute trial of a single i.v. dose of $0.3 \mathrm{mg} / \mathrm{kg}$ has previously been reported Longer term effects are unknown.

Twelve MND patients aged 34-66 years were infused $0 \cdot 1-0 \cdot 35 \mathrm{mg} / \mathrm{kg}$ i.v. over $2-6$ hours daily or at longer intervals for up to ten weeks, after baseline assessments. Eight patients with bulbar symptoms showed improvement in speech with objective changes in tongue, lip and jaw movements, lasting about one week after cessation of therapy. Five had improvement in vital capacity and four in maximal respiratory pressures. Two had moderate improvement in swallowing. Two further cases showed deterioration in respiratory parameters, one had significant side effects, the other was asthmatic. Dynamometry in eighty-five muscles showed improvement in ten in five patients and deterioration in eleven in five patients for up to five days after cessation of therapy. Some activities of daily living improved in three of six tested patients. Cramps improved for up to eight weeks in five patients. The drug was useful for bulbar symptoms but its effect on muscle force was not predictable. Careful individual adjustment of the dose was required. Long term studies are indicated.
THERMAL THRESHOLD TESTING IN DIABETIC NEUROPATHY

Al Weir, G Jamal, S Hansen, JP Ballantyne. Institute of Neurological Sciences, Glasgow

The criteria for diagnosis of diabetic peripheral neuropathy are not universally agreed. Dyck $^{1}$ has sought to investigate this problem using a combination of clinical scoring methods, automated sensory testing, electrophysiology and neuropathology. We have also completed a comprehensive study of 73 diabetics in whom neuropathy was diagnosed or suspected clinically.

Dyck detected abnormalities of thermal cooling in 13/23 (56\%) using his computer assisted sensory examination and concluded that thermal sensation was the least sensitive in the detection of abnormality when compared with touch/pressure and vibration. We have used the Glasgow designed thermal threshold testing technique and detected abnormality in $69 / 73(95 \%)$ of our patients. There is therefore a considerable discrepancy between the techniques.

We have compared published results from the two techniques for absolute values and inter-individual variablity. Secondly, w记 have modified our equipment to simulate the $\vec{\sigma}$ method and procedure used by Dyck and other groups to determine thermal thresh olds. In each case our technique produces lower absolute thresholds and propor tionally much smaller standard deviations This decreases the overlap between the ab normal and normal populations to a ming imum.

We conclude that dogmatic statements as to the usefulness of testing certain modalities of sensation in detection of neuropathy must be qualified by the type of technique used. With our technique thermal threshold testing detects abnormality in $95 \%$ of diabetics in whom neuropathy is suspected.

${ }^{1}$ Dyck PJ, Karnes JL, Dambe J, O'Brien P, Service FJ Clinical and neuropathological criteria for the diagnosis and staging of diabetic polyneuropathy.

Brain: 1985:108, 861-880

SPINAL CORD STIMULATION AND THE RELIEF OF CHRONIC PAIN

TH Koeze, AC deC Williams, S Reiman, ES Watkins. The London Hospital Medical College, London

Spinal cord stimulation has been used for the relief of chronic pain for nearly twenty years. Long term results have not been encouraging. However, many early studies included patients with a history of drug abuse and psychotic illness. Most were done by postal surveys or retrospective data collection from 
clinical records. We studied 26 patients with chronic intractable pain without a history of drug abuse or psychotic illness who received spinal cord stimulation for a median period of 28 months. The patients were evaluated by structured videotaped interviews with psychologists not involved in the patients care. In addition estimates of pain relief were obtained from clinicians involved in the patient's care and from close relatives or friends. Information about general activities and drug usage was also collected. At the time of the interview half of the patients were receiving $50 \%$ or better relief of their pain. There was a decrease in the use of analgesic medication and increased domestic and leisure activity. The "costs" of this result included repeated visits to outpatient clinics for detection and repair of stimulator faults.

RADIOLOGICAL SUPPORT FOR THE PRIMARY ROLE OF OCCIPITAL DYSPLASIA IN THE ADULT CHIARI MALFORMATION

W Schady, RA Metcalfe, P Butler. Departments of Neurology and Neuroradiology, Manchester Royal Infirmary, Manchester

The reason for cerebellar herniation in the adult Chiari type 1 malformation (ACM 1) is not known. It has been postulated that it is secondary to a reduction in size of the bony posterior fossa, although the reported incidence of platybasia and basilar invagination in ACM 1 is only $20-30 \%$. In order to test this hypothesis detailed surface area, angular and linear measurements were undertaken on plain radiographs of 32 symptomatic patients (mean age 39 years) with myelographically proven primary ACM 1 .

Basal and Boogard's angles were significantly larger in patients than in controls. The clivus was shorter, Klaus' level index was smaller and the area of the bony posterior fossa in the saggital plane was reduced in patients compared with controls, even after correction for unequal total skull size. By the use of discriminant analysis $68 \%$ of patients were accurately predicted to belong to the patient group and $94 \%$ of controls were identified as such.

These results show that over two-thirds of patients with ACM 1 have recognisable occipital dysplasia. Furthermore, there was an inverse relationship between the size of the posterior fossa and the degree of cerebellar herniation. This suggests that the bony anomaly is primary and that tonsillar descent occurs as a result of crowding out of the cerebellum.
PRE-BYPASS HYPOCAPNIA IS CORRELATED WITH THE POST-OPERATIVE APPEARANCE OF SIGNS OF OCULAR HYPOPERFUSION IN PATIENTS UNDERGOING CORONARY ARTERY BYPASS SURGERY

M Nevin, A Colchester, J Pepper, S Adams, R Humphrey, A Davies. St George's Hospital, London

Thirty patients undergoing coronary artery bypass surgery had detailed neurological, ophthalmological and psychometric assessment pre-operatively and again on the 3rd and 7 th post-operative day. Pre-operatively arterial and jugular bulb venous pressures and gas tensions were monitored frequently but the surgeon and anaesthetist were not informed of these results.

In 15 patients (Group I) expired gas $\mathrm{CO}_{2}$ monitoring was not used by the anaesthetist who referred to intermittent blood gas samples according to routine practice. Seven of the patients developed new eye signs postoperatively (Group Ia): five had retinal changes consistent with ocular hypoperfusion and two showed a reduction in visual acuity without retinal changes. For Group Ia the mean $\mathrm{PaCO}_{2}$ immediately prior to bypass was $27 \cdot 1 \mathrm{~mm} / \mathrm{Hg}$. A significant psychometric deficit was found in five patients.

The other eight patients in Group I did not have eye signs (Group Ib). They had a mean $\mathrm{PcCO}_{2}$ prior to bypass of $36.0 \mathrm{mmHg}$ and only three showed a significant psychometric deficit post-operatively.

In the remaining 15 patients (Group II) the anaesthetist used end-tital $\mathrm{CO}_{2}$ monitoring and took special care to maintain normocapnia. The only post-operative eye signs observed were glaucomatous changes in the one patient, although six Group II patients developed a psychometric deficit. The mean $\mathrm{PaCO}_{2}$ prior to bypass was 37.4 $\mathrm{mm} / \mathrm{Hg}$.

Statistical analysis showed a highly significant difference $(p<0.001)$ between the pre-bypass $\mathrm{PaCO}_{2}$ values in the patients with eye signs (Group Ia) and those without eye signs (Groups Ib and II combined). There was no evidence in any of the patients of retinal microembolisation.

BRADYPHRENIA IN PARKINSON'S DISEASE AND PSYCHOMOTOR RETARDATION IN DEPRESSION: AN EXPERIMENTAL STUDY

AJ Lees, D Rogers, E Smith, M Trimble, GM Stern. The National Hospitals for Nervous Diseases, London

Thirty newly diagnosed patients with Parkinson's disease showed motor and cognitive slowing, or bradyphrenia, on a computerise digit symbol substitution test, related to mo tor and cognitive impairment. Thirty pa: tients with primary depressive illness showed comparable deficits, related to motor im pairment. Retesting 12 of the Parkinsonian? patients after treatment with dopaminergiष agonists, and 12 of the depressed patients? showed improvement in affective im pairment. It is suggested that bradyphrenia in Parkinson's disease and psychomotor re tardation in depressive illness are closely re lated and that impairment of dopaminergic systems may be involved in both.

BRADYKINESIA-A FAILURE OF COROLLARY DISCHARGES?

AP Moore. Institute of Neurolologica $\overrightarrow{5}$ Sciences, Glasgow

The pathophysiological mechanism of bra. dykinesia is unclear. It is thought to be due to a failure of the basal ganglia to "executẹ learned motor plans". This may be a purelyor motor abnormality but many authors havésuggested that sensory features are im $N$ portant. This experiment was an attempt $10-\vec{V}$ distinguish between the two possibilities. $\frac{O}{\mathbb{D}} \vec{\sigma}$

With eyes closed, patients with asym-o metric bradykinesia (1) matched the morements of one guided (reference) arm with the other arm and (2) matched the movementsof both arms in self guided actions.

Movements were active not passive, $\frac{1}{3} \mathrm{~d} 3$ were not rapid. Controls accurately performed both tasks. Patients regularly inlowed the bradykinetic reference arm to lag, even when the good arm was matching the more bradykinetic reference arm. The accuracy of matching was correlated with the symmetry of bradykinesia. Patients thus thought the bradykinetic limb moved furthero than it had.

Parkinsonian patients with drug induced $\stackrel{\mathbb{Q}}{\varrho}$ unilateral dyskinesia, patients with idio- $\overrightarrow{\vec{A}}$ pathic unilateral dystonia, and patients with weakness of one arm moved the abnormal limb further.

It is suggested that bradykinesia could be caused by reduction in corollary discharges, and dyskinesia and dystonia result from exaggeration of corollary discharges.

DYSKINESIA IN MPTP-TREATED PRIMATES ON LONGTERM LEVODOPA THERAPY

CE Clarke, S Boyce, AR Crossman, MA응 Sambrook. Experimental Neurology Group, Medical School, Manchester

The administration of MPTP to non-human primates produces a Parkinsonian syndrome

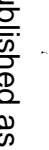


comparable to idiopathic Parkinson's disease in man. Using this animal model the effect of long-term levodopa administration has been examined, and, in particular, whether the complications of such treatment in Parkinson's disease can be reproduced. After 4-8 weeks treatment with therapeutic doses of levodopa all animals developed peak-dose choreiform movements affecting the lower limbs. Despite a constant dose regime over a further 6 months, the dyskinesia became more severe and extensive involving the upper limbs and face. A second group of MPTP-treated animals did not receive regular levodopa therapy and the severity of the Parkinsonian features remained stable over at least 6 months.

Since the Parkinsonian syndrome remained constant in the non-levodopa- treated animals, the onset and progress of dyskinesia in the treated animals must relate to levodopa treatment rather than disease progression. This confirms the results of clinical trials which suggest that the introduction of levodopa therapy in Parkinson's disease should be delayed and used at the lowest possible dose.

THE NATURAL HISTORY OF UNTREATED EPILEPSY

RDC Elwes, AL Johnson, EH Reynolds. Department of Neurology, Kings College Hospital, London and MRC Biostatistics Unit, Cambridge

Recent studies suggest that the prognosis for newly referred epileptic patients treated with carefully monitored monotherapy is very good. What is lacking is any information about the natural history and prognosis of untreated epilepsy as, for ethical reasons, such studies have never been undertaken. A limited view of natural history can be obtained by detailed retrospective analysis of untreated seizures in patients referred for the first time to a hospital clinic. Of 281 patients with 2-5 untreated tonic clonic seizures the attacks can be accurately dated in 183 patients. Overall there was a progressive decline in seizure intervals between successive seizures. In only $20 \%$ of instances did the interval between any successive seizures in crease. Our observations provide some support for Gowers' view that seizures may beget further seizures and for the need for early effective treatment. 